\title{
Article \\ Construction and Characterization of Representations of SU(7) for GUT Model Builders
}

\author{
Daniel Jones and Jeffery A. Secrest *
}

check for updates

Citation: Jones, D.; Secrest, J.A. Construction and Characterization of Representations of SU(7) for GUT Model Builders. Symmetry 2021, 13, 1044. https://doi.org/10.3390/ sym13061044

Academic Editors: Andrea Lavagno and Maxim Yu. Khlopov

Received: 5 April 2021

Accepted: 28 May 2021

Published: 9 June 2021

Publisher's Note: MDPI stays neutral with regard to jurisdictional claims in published maps and institutional affiliations.

Copyright: (c) 2021 by the authors. Licensee MDPI, Basel, Switzerland. This article is an open access article distributed under the terms and conditions of the Creative Commons Attribution (CC BY) license (https:// creativecommons.org/licenses/by/ $4.0 /)$.
Department of Physics and Astronomy, Armstrong Campus, Georgia Southern University, Savannah, GA 31419, USA; dj03057@georgiasouthern.edu

* Correspondence: jsecrest@georgiasouthern.edu

\begin{abstract}
The natural extension to the SU(5) Georgi-Glashow grand unification model is to enlarge the gauge symmetry group. In this work, the $\mathrm{SU}(7)$ symmetry group is examined. The Cartan subalgebra is determined along with their commutation relations. The associated roots and weights of the $\mathrm{SU}(7)$ algebra are derived and discussed. The raising and lowering operators are explicitly constructed and presented. Higher dimensional representations are developed by graphical as well as tensorial methods. Applications of the SU(7) Lie group to supersymmetric grand unification as well as applications are discussed.
\end{abstract}

Keywords: GUT; grand unified theories; quarks; leptons; gauge symmetry breaking; lie algebra; gauge group

\section{Introduction}

Symmetry is central to understanding the structure of nature at the largest and smallest scales. The current theory of elementary particles and their interactions is the highly successful Standard Model of particle physics [1-7]. This model is governed by the $\mathrm{SU}(3)_{C} \times \mathrm{SU}(2)_{L} \times \mathrm{U}(1)_{Y}$ local gauge symmetry groups that describe the color symmetries of the strong interaction and weak isospin and hypercharge symmetries of the electroweak interaction. The Standard Model has amassed a number of stunning successes since its inception such as predictions of the weak mixing angle $[8,9]$, the existence of weak neutral currents [10,11], and the generation of mass via the Higgs mechanism [12,13]. However the Standard Model hints at deeper physics suggesting that it is but a low-energy effective theory. The Standard Model is plagued by a relatively large number of arbitrary parameters (the quark and neutrino mixing angles, fermionic coupling constants, Higgs potential parameters, etc), the mystery of electric charge quantization, and a number of other nagging questions on the nature of neutrino and dark matter properties. Grand Unified Theories (GUTs) [14-18] are focused on embedding the Standard Model gauge groups into a larger gauge symmetry group.

In general, grand unification leads to a number of aesthetically pleasing results. Some of these include:

- $\quad$ low energy gauge couplings are unified into a single gauge coupling at the GUT scale;

- a simplified explanation as to the origin of electric charge and quantization;

- $\quad$ anomaly constraints linking quarks and leptons of a single generation together;

- $\quad$ the particle spectrum structure is simplified, fitting into minimal multiplets; and

- introduces an elegant explanation to the observed cosmological baryon excess of the universe.

Grand unified theories also lead to explicit predictions that can be tested experimentally. Some of these predictions include:

- $\quad$ baryon and lepton number violation, most famously leading to proton decay and neutron-anti-neutron oscillations; 
- $\quad$ the existence of magnetic monopoles connected with electric charge quatization;

- $\quad$ prediction of the weak mixing angle, $\sin ^{2} \theta_{W}$, at low energies; and

- mass relationships between leptons and quarks.

It should be noted, the goal of grand unified theories is the ultimate unification into a single paradigm of the strong, weak, and electromagnetic interactions with the elementary fermions, quarks and leptons. In addition to this framework, GUTs have several cosmological applications that may provide deeper insights into the evolution of the universe from the earliest epochs and their consequences to the observed universe today. Questions associated with understanding the flatness, isotropy, and homogeneity of the currently observed universe can be reconciled by considering GUT symmetry breaking in an early metastable phase where a scalar field is trapped in a metastable minimum [19-21]. The potential associated with this scalar field then drives the superluminal expansion of the universe. Fluctuations in this scalar field will contribute to the generation of the large scale structure of the universe observed today [22-25]. This induced exponential, or quasi-exponential, expansion of the universe in turn then benefits GUT models that produce copious amounts of topological stable particles that are not observed such as magnetic monopoles, domain walls, and/or cosmic strings by diluting them out. Finally, questions associated with the matter-anti-matter asymmetry of the universe can be naturally explained via new GUT interactions that inherently violate baryon and lepton number conservation leading to baryogenesis at the highest energies and at the earliest times [26-29].

The SU(7) group has found a wide range of applications in many non-supersymmetric GUTs where the Standard Model has been embedded inside as a subgroup. It has been used in flavor unification [30] to provide an explanation as to why there are multiple fermionic generations. The use of the $\mathrm{SU}(7)$ spinor representation allows for easy extension to an $\mathrm{O}(14)$ theory. Exotically charged quarks are predicted, as is a low mass magnetic monopole that does not conflict with big bang cosmology. Another effort has been the technicolor GUT [31], in which the SU(5) Georgi-Glashow model is combined with an additional $\mathrm{SU}(2)_{T C}$ technicolor symmetry [32] in which $\mathrm{W}$ and $\mathrm{Z}$ bosons acquire mass via a new gauge interaction. Here $\mathrm{SU}(7) \rightarrow \mathrm{SU}(5)_{S} \times \mathrm{SU}(2)_{L} \times \mathrm{U}(1)_{Y}$, where $\mathrm{SU}(5)_{S}$ then breaks into $\mathrm{SU}(3)_{C} \times \mathrm{SU}(2)_{T C} . \mathrm{SU}(7)$ is also often embedded into $\mathrm{SO}(14)$ models [33] as an intermediate breaking chain $\mathrm{SO}(14) \rightarrow \mathrm{SU}(7)$. Four families of fermions are predicted with the same quantum numbers, but distinct structures. In this model, the flavor problem is given a satisfying answer under the assumed framework in which the fermions are postulated to be composite. It should be noted that the $\mathrm{SU}(7)$ group accommodates the left-right symmetric Pati-Salam model, $\mathrm{SU}(3)_{C} \times \mathrm{SU}(2)_{L} \times \mathrm{SU}(2)_{R} \times \mathrm{U}(1)_{Y}$ where it has been applied to studying the muon $g-2$ anomaly [34] along with so-called 331 models, $\mathrm{SU}(3)_{C} \times \mathrm{SU}(3)_{L} \times \mathrm{U}(1)_{Y}$ as a natural avenue for describing the existence of three generations predicated upon anomaly cancellation [35].

The SU(7) symmetry group has been applied to supersymmetric (SUSY) GUTs where there is an additional proposed symmetry between fermions and bosons. One of the most appealing aspects of SUSY GUTs are the lack of accidental symmetries such as those leading to global conservation laws like lepton and baryon number conservation. SUSY orbifold GUTs have the additional appealing aspect that they occur in a higher dimensional space but the gauge symmetry reduces to an effective 4-dimensional theory of the minimal supersymmetric standard model (MSSM). In these models, symmetry breaking is typically associated with boundary conditions in extra-dimensions. This allows the models to retain many of the desirable features of grand unification. The SU(7) group has been consider as a SUSY 5-dimensional oribifold GUT effective theory below some generic herotic string scale where gauge-Higgs unification is present. In this type of model, the Higgs scalar is an extra-dimensional component of the gauge field [36]. In another SUSY SU(7) model, the hierarchies amongst the particle generations are generated by the hierarchy of symmetry breaking scales of the gauge group. Here $\mathrm{SU}(7) \rightarrow \mathrm{SU}(5) \times \mathrm{SU}(2)^{\prime}$ where the $\mathrm{SU}(2)^{\prime}$ group breaks into two, relatively smaller scales of the first and second particle generations [37]. 


\section{The SU(7) Group}

The special unitary group of 7 dimensions, $\mathrm{SU}(7)$, is the Lie group of all the $7 \times 7$ unitary matrices, $U$, with unit determinant. This can be formally written as,

$$
\mathrm{SU}(7)=\left\{U \in \mathrm{GL}\left(\mathbb{C}^{7}\right): U^{+} U=\mathbb{I}_{7}, \operatorname{det} U=1\right\}
$$

where GL is the general linear group, $\mathbb{I}_{7}$ is the $7 \times 7$ identity matrix, and $U^{+}$indicates the hermitian conjugate of the transformation matrix $U$. This transformation group preserves the hermitian product invariant under rotations in the complex space $\mathbb{C}^{7}$.

The fundamental representation of $\mathrm{SU}(7)$ is a vector with 7 complex elements, sometimes referred to as the 7 ,

$$
\phi=\left(\begin{array}{c}
\phi_{1} \\
\phi_{2} \\
\vdots \\
\phi_{7}
\end{array}\right)
$$

that transforms as,

$$
\phi \rightarrow U \phi
$$

where $U$ are the $7 \times 7$ uni-modular unitary matrices described above. The $\mathrm{SU}(7)$ group, by virtue of being a Lie group, is also a differential manifold that one can Taylor series expand around the identity and thus generate any other elements in the group. The infinitesimal transformation can be written as,

$$
d U=\sum_{n=1}^{48}\left(\mathbf{1}+i T_{n} d \theta_{n}\right)
$$

where $T_{n}$ are the normalized group generators described in Section 3 and $\theta_{n}$ are variable parameters that are real and continuous. Any finite transformation can be built up from repeated application of the infinitesimal transformations,

$$
U=\lim _{k \rightarrow \infty}\left(\mathbf{1}+i \frac{T_{n} \theta_{n}}{k}\right)^{k}=\exp \left(-i \sum_{n=1}^{48} \theta_{n} T_{n}\right) .
$$

\section{Generators}

Associated with the Lie group SU(7) is the Lie algebra $\mathfrak{s u}(7)$ that obeys the commutator relationship,

$$
\left[T_{i}, T_{j}\right]=i \sum_{k}^{48} f_{i j}^{k} T_{k}
$$

where $T_{i}$ are the normalized generators and $f_{i j}^{k}$ are known as the structure constants. There are forty-eight $7 \times 7$ traceless and hermitian matrices that are the generators for the $\mathrm{SU}(7)$ group. These generators are not unique. One method of determining the generators is to create a vector space spanned by the matrix,

$$
\left(\begin{array}{cccc}
a_{1} & a_{12}-i b_{12} & \cdots & a_{17}-i b_{17} \\
a_{12}+i b_{12} & a_{2} & \cdots & \vdots \\
\vdots & \vdots & \ddots & \\
a_{17}+i b_{17} & & & a_{7}
\end{array}\right)
$$

where $a_{i}$ and $b_{i}$ are real numbers. It should be noted that there is a constraint that the sum of the diagonal elements must equal zero in order to fulfil the definition of the $\mathrm{SU}(7)$ group.

The explicit form of the generators of the Lie algebra of $\mathrm{SU}(7)$ are not unique though the structure constants are unique. An explicit form of the the non-normalized generators 
and the structure constants have been reproduced from reference [38] and can be found in Appendices A.1 and A.2 respectively.

\section{Representations of SU(7)}

A representation of a group $G$ over an $n$-dimensional vector space $V$ is a map

$$
\pi: G \rightarrow \mathrm{GL}(V)
$$

of group elements $g$ to a space of linear operators $D(g)$. This map ensures that the identity $e$ is mapped to the identity in $\mathrm{GL}(V)$,

$$
D(e)=I_{n}
$$

where $I_{n}$ is the $n \times n$ identity matrix. Group composition is preserved such that,

$$
D\left(g_{1}\right) D\left(g_{2}\right)=D\left(g_{1} g_{2}\right)
$$

for any $g_{1}$ and $g_{2}$ in $G$. More specifically, the topics of interest are representations of $\mathrm{SU}(7)$ corresponding to group homomorphisms,

$$
\pi: \mathrm{SU}(7) \rightarrow \mathrm{GL}(n, \mathbb{C})
$$

where $\mathrm{GL}(n, \mathbb{C})$ is the general linear group of $n \times n$ invertible, complex-valued matrices. The dimension of the representation is given by $n$. Group elements in any $n$-dimensional representation can still be found via the relation in Equation (4), only the generators $T_{k}$ are replaced by their $n \times n$ representational counterparts. Irreducible representations of $\mathrm{SU}(7)$ are constructed with traceless tensors with $m$ upper and $n$ lower indices,

$$
\phi_{j_{1}, \ldots, j_{n}}^{i_{1}, \ldots, i_{m}}
$$

Each index individually takes on integer values from 1 to 7 and transforms independently of the other indices. A tensor is antisymmetric if it changes sign on the interchange of two indices such that,

$$
\phi^{i j}=-\phi^{j i}
$$

otherwise it is symmetric. Whether two indices are symmetric or not directly determines the dimension of the representation furnished by that tensor. Indices enclosed in square brackets $[i j]$ will indicate that they are antisymmetric, whereas curly brackets $\{i j\}$ will indicate they are symmetric.

As mentioned previously, the fundamental representation is contained in a vector that transforms as a 7-dimensional, single index tensor, referred to as the 7. The trivial representation, the 1, is a scalar that is invariant under all transformation. Examples of higher dimensional, irreducible representations of $\mathrm{SU}(7)$ furnished by traceless tensors with more indices include:

- $\phi_{j}^{i}$

- $\phi^{[i j]}$ and $\phi^{\{i j\}}$

- $\phi_{k}^{[i j]}$ and $\phi_{k}^{\{i j\}}$

- $\quad \phi^{[i j k]}$ and $\phi^{\{i j k\}}$

where the above is not an exhaustive list. If the tensor $\phi$ were not traceless, it could be decomposed into the direct sum of a traceless tensor $\phi^{\prime}$ and the trace of the original tensor $\operatorname{Tr} \phi$. The complex conjugate of a representation is defined by the interchange of the upper and lower indices such that,

$$
\left(\phi_{j_{1} \ldots j_{n}}^{i_{1} \ldots i_{m}}\right)^{*} \equiv \phi_{i_{1} \ldots i_{m}}^{j_{1} \ldots j_{n}} .
$$

A representation in which the number of upper indices matches the number of lower indices is said to be real or self-adjoint, and is equivalent to its own conjugate. The remainder are 
referred to as complex representations. The dimension of a tensor representation is given by the following formula,

$$
\operatorname{dim} \phi=\left(\operatorname{dim} \phi^{i_{1}, \ldots, i_{k}}\right)\left(\operatorname{dim} \phi_{j_{1}, \ldots, j_{l}}\right)-\operatorname{dim}(\operatorname{Tr} \phi) .
$$

The first term on the right-hand side is the product of the dimension of the upper indices with the dimension of the lower indices. The second term is the dimension of the trace. The trace is subtracted out as irreducible representations are furnished by traceless tensors only. The dimension of a representation and its conjugate take on the same numerical value.

Special diagrams known as Young tableau can also be utilized to find the dimension of a particular representation and, as discussed in Section 5, the decomposition of direct products between representations. The English notation of Young tableau [39] is employed. The fundamental representation of $\mathrm{SU}(7)$ and its conjugate, 7 and $\overline{7}$, are expressed using tableaux as,

and

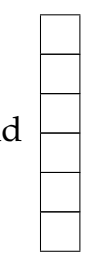

The diagrams can be written in terms of tableau sequences $(1,0,0,0,0,0)$, and $(0,0$, $0,0,0,1)$ respectively. This written notation for a Young tableau in $\mathrm{SU}(7)$ is given by a 6-component sequence $\left(n_{1}, n_{2}, n_{3}, n_{4}, n_{5}, n_{6}\right)$, where each component is a non-negative integer. The position of the right-most nonzero entry in the list denotes the number of rows in the tableau. There can not be more than 7 rows in a column, and a column with 7 rows denotes the trivial representation. The integer at the $i$-th position in the sequence denotes how many more columns that row $i$ contains than row $i+1$. The conjugate of a representation is found by reversing the order of the entries of the sequence.

Using Young tableaux, the dimension $N$ of a representation in $\mathrm{SU}(7)$ is given by the formula,

$$
N=\prod_{i=1}^{n}\left(\frac{D_{i}}{h_{i}}\right)
$$

where $n$ is total number of blocks in that specific tableau. The index $i$ is counted on the tableau blocks from left-to-right, and top-to-bottom. $D_{k}$ is defined as 7 plus the number of rightward steps and minus the number of downward steps from the $i=1$ to $i=k$ position. The hook length, $h_{k}$, is defined as the sum of the number of boxes to the right of the box at $i=k$ and below it, plus 1 . The indexing, $D_{i}$ values, and $h_{i}$ values, respectively, are shown below in example tableau,

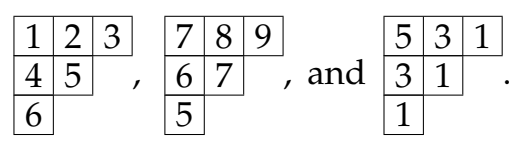

An alternative formula for calculating dimensions of Young tableaux for $\mathrm{SU}(7)$ is,

$$
N=\prod_{i=0}^{5}\left(\prod_{j=1}^{6-i} \frac{\left(\sum_{k=j}^{j+i} n_{k}\right)+i+1}{i+1}\right)
$$

where each value of the index $i$ runs through a full set of the $j$ index.

The tensor $\phi_{j}^{i}$ denotes the $\mathbf{4 8}$, and is the adjoint representation. It is the smallest, nontrivial real representation of the group, the diagram for which is shown below, 


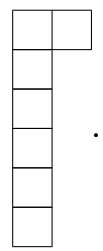

The antisymmetric and symmetric tensors, $\phi^{[i j]}$ and $\phi^{\{i j\}}$, are the $\mathbf{2 1}$ and $\mathbf{2 8}$ as seen below,

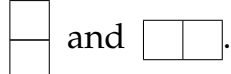

The tableau sequences $(2,0,0,0,0,0)$ and $(0,0,0,0,0,2)$, corresponding to $\varphi^{\{i j\}}$ and $\varphi_{\{i j\}}$ are written as,

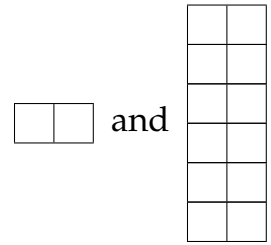

The tableau sequences $(2,0,0,0,0,1)$ and $(1,0,0,0,0,2)$ correspond to $\varphi_{k}^{\{i j\}}$ and $\varphi_{\{i j\}^{\prime}}^{k}$ and are written as,

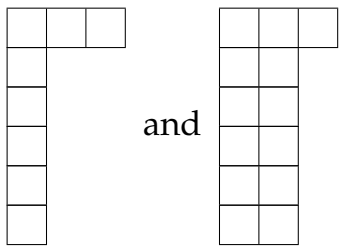

The tableau sequences $(0,2,0,0,0,1)$ and $(1,0,0,0,2,0)$ correspond to $\varphi_{k}^{[i j]}$ and $\varphi_{[i j]}^{k}$, and are written as,

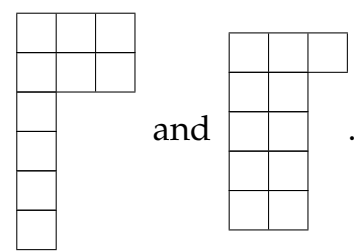

\section{Decomposition}

Larger representations may be constructed via the direct products of irreducible representations of $\mathrm{SU}(N)$. As such, they can by decomposed into a direct sum of irreducible representations. Using the tensor approach the direct product is defined as,

$$
\phi_{n}^{m} \otimes \phi_{n^{\prime}}^{m^{\prime}} \equiv \eta_{n+n^{\prime}}^{m+m^{\prime}} .
$$

A traceless tensor with $m$ upper and $n$ lower indices is in a product with another traceless tensor with $m^{\prime}$ upper and $n^{\prime}$ lower indices. This is equivalent to a single tensor with $m+m^{\prime}$ upper and $n+n^{\prime}$ lower indices. This tensor is not always irreducible and may contain a non-zero trace element. This product is then decomposed into a direct sum in which both symmetric and antisymmetric combinations of indices are considered, as well as trace terms.

Decomposing the product $7 \times 7$ using the tensor approach,

$$
\phi^{i} \otimes \phi^{j}=\phi^{\{i j\}} \oplus \phi^{[i j]} .
$$


Both a symmetric and antisymmetric combination of the combined indices have been considered. The lack of any lower index makes the product traceless by default. Young tableau can be utilized more efficiently for decomposition. Using tableau rules for products, the same product can be written as,

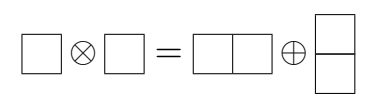

which matches the tensor approach. In terms of their dimensions, the product can be expressed as

$$
7 \otimes 7=28 \oplus 21 .
$$

Taking the product $\mathbf{7} \times \overline{\mathbf{7}}$ using tensor notation results in the equation

$$
\phi_{i} \otimes \phi^{j}=\phi_{i}^{j} \oplus \phi .
$$

Using the tableaux, a matching result is achieved,

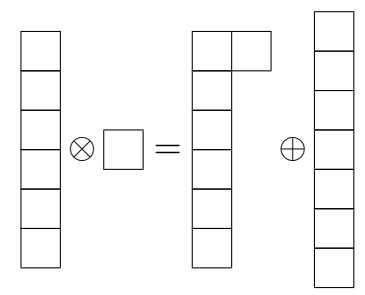

Both methods agree on the decomposition of the product,

$$
7 \otimes \overline{7}=48 \oplus 1
$$

into a sum of the adjoint and singlet. Note that no considerations regarding index symmetry had to be taken. However, the trace term represented by the singlet had to be accounted for. These products play a pivotal role in GUTs when building up other representations using products involving the fundamental representation and its conjugate.

\section{Raising and Lowering Operators}

In quantum theory it is standard practice to utilize $\mathrm{SU}(2)$, where the generators undergo a transformation to the polar basis and are renamed raising and lower operators, to describe the energy states of the quantum harmonic oscillator problem. It is beneficial to perform an analogous transformation here with the $\mathrm{SU}(7)$ generators. The forty-eight generators of $\mathrm{SU}(7)$ are defined as,

$$
T_{a}=\frac{1}{2} \lambda_{a}
$$

where $a=1, \ldots, 48$, and the $\lambda_{a}$ are the SU(7) extensions of the Pauli [40] and Gell-Mann [41] matrices. The generators obey the chosen trace normalization condition,

$$
\operatorname{Tr}\left(T^{a} T^{b}\right)=\frac{1}{2} \delta^{a b} .
$$

$\mathrm{SU}(7)$ has six simultaneously diagonalizable generators, which will be discussed in Section 7. The remaining 42 generators are defined as linear combinations of the off-diagonal matrices,

$$
V_{ \pm \alpha}=\frac{1}{\sqrt{2}}\left(T^{a} \pm i T^{b}\right)
$$

where $\alpha=1,2, \ldots, 21$ and $V_{ \pm \alpha} \in G L(n, \mathbb{R})$. Here, $n$ is dependent on the representation. $T_{a}$ are the 21 symmetric generators, and $T_{b}$ are their antisymmetric counterparts. A pair of raising and lowering operators with indices $\pm \alpha$ can be related by, 


$$
V_{-\alpha}=\left(V_{\alpha}\right)^{T} .
$$

The explicit forms of the raising and lowering operators were calculated and cataloged in Table A3 in Appendix A, where $E_{i j}$ is a $7 \times 7$ matrix with a 1 in the $i j$ position and 0 's elsewhere.

\section{Cartan Generators}

The remaining generators are simultaneously diagonalizable and form the Cartan subalgebra of the group. This is the maximal Abelian subalgebra of the group, and it is uniquely found. These elements are called Cartan generators and are denoted by an $H^{i}$ where $i=1, \ldots, 6$. They are real-valued, Hermitian matrices that obey the commutation relations

$$
\left[H^{i}, H^{j}\right]=0
$$

for all combinations of $i$ and $j$. They satisfy the trace normalization in Equation (20). The Cartan generators have been tabulated in Table A5 in Appendix A. Symmetry breaking is an important feature of GUTs. The exact values of the components of the Cartan generators will change depending on the desired breaking pattern. Irrespective of any desired breaking pattern they will always be diagonalized, traceless, and obey a trace normalization condition. In this work they have not been formulated with any particular breaking patterns in mind.

The Cartan subalgebra holds great significance in model building, as the operators corresponding to physical observables can be expressed as linear combinations of the commuting generators in weight space. Common examples would be the operators corresponding to electric charge or the third component of weak isospin.

\section{Weights}

Each irreducible representation has its own set of states, the number of which is equal to the dimension of the representation. For example, the fundamental representation has seven states $\left|\omega_{j}\right\rangle$, where $j=1, \ldots, 7$. They are six component vectors called weight vectors, and their components are referred to as weights. In the fundamental representation, the weights are the eigenvalues of the Cartan generators acting upon the weight vectors,

$$
H^{i}\left|\omega_{j}\right\rangle=h^{i}\left|\omega_{j}\right\rangle .
$$

In actual application, the weights represent the various quantum numbers of each particle state in a given representation. Since the Cartan generators have been diagonalized, the $i$-th component of the $j$-th weight vector $\omega$ is given by,

$$
\left[w_{j}\right]^{i}=\left[H^{i}\right]_{j, j} .
$$

The weight vectors of the adjoint representation, $\left|X_{n}\right\rangle$, where $n=1, \ldots, 48$, are characterized by the forty-eight generators. $X_{n}$ are the $48 \times 48$ matrix representations of both the raising and lowering operators and Cartan generators. So let $n=1, \ldots, 42$ be the raising and lower operators, and let $n=43, \ldots, 48$ denote the Cartan generators. The states of the adjoint are orthogonal,

$$
\left\langle X_{n} \mid X_{m}\right\rangle=\delta_{n m}
$$

and satisfy a completeness relation,

$$
\sum_{n=1}^{48}\left|X_{n}\right\rangle\left\langle X_{n}\right|=I .
$$

Using Equation (27), it is determined that the action of the raising and lowering operators on the states is given by,

$$
X_{n}\left|X_{m}\right\rangle=\left|\left[X_{n}, X_{m}\right]\right\rangle .
$$


Using Equations (28) and (23), it can be shown that each weight vector corresponding to a Cartan generator is the zero vector. The weights of the other states are given by the same eigenvalue equation in Equation (24) such that,

$$
X_{i}\left|X_{n}\right\rangle=\alpha\left|X_{n}\right\rangle
$$

for $i=43, \ldots, 48$. The weight vectors of the adjoint representation have important properties, to be discussed in Section 9, and as such are referred to explicitly as root vectors.

\section{Roots}

While the relations detailed in Section 8 are derived using the adjoint representation, they can be applied to the fundamental representations to explore the root system of the algebra. The root system is a set of vectors in a Euclidean vector space with particular geometric properties which will be explored in detail in this section.

The components of the root vectors can be calculated by combining Equations (28) and (29). The result is the following commutation relationship,

$$
\left[H^{i}, V_{ \pm \alpha}\right]= \pm \alpha^{i} V_{ \pm \alpha} .
$$

Here $\alpha^{i}$ is the component of the corresponding root vector and is known as a root. Root vectors are denoted by $v_{ \pm \alpha}$, where $\alpha=1, \ldots, 21$. The root vectors are unitary with our chosen normalization, i.e., $\left\|v_{\alpha}\right\|=1$ for all $\alpha$. Here $\left\|v_{\alpha}\right\|$ is defined as the square root of the Euclidean inner product of $v_{\alpha}$ with itself in six dimensions. The roots of a raising operator and its corresponding lowering operators differ only by a minus sign, so $v_{-\alpha}=-v_{\alpha}$. Since half of the roots are copies of the other half differing only by a minus sign, this means that the discussion can be limited to a subset of vectors without any loss. This subset of root vectors are called the positive roots and in this work have been defined to be the root vectors in which the first nonzero component is nonnegative.

Using this relationship and Equation (23) in the adjoint gives,

$$
X_{\alpha}\left|X_{-\alpha}\right\rangle=|0\rangle
$$

which implies that it can be written as a linear combination of the states corresponding to the Cartan generators, giving the relationship

$$
\left[V_{\alpha}, V_{-\alpha}\right]=\alpha_{i} H^{i}
$$

where Einstein summation convention [42] is employed and $\alpha_{i}=\alpha^{i}$. In general, the commutator of two raising and lowering operators is,

$$
\left[V_{\alpha}, V_{\beta}\right]=N_{\alpha \beta} V_{\alpha+\beta}
$$

where $N_{\alpha \beta} \in \mathbb{R}$. If $v_{\alpha}+v_{\beta}$ does not result in another root vector, then $N_{\alpha \beta}=0$. Otherwise $v_{\alpha}+v_{\beta}$ is another root. Since any $V_{\alpha}$ self commutes this implies that if $v_{\alpha}$ is a root vector then $k v_{\alpha}$ is also a root vector which in turn implies that $k$ can only take on values of 0 or \pm 1 .

Any positive roots that cannot be expressed as a linear combination of two other positive roots are defined as simple roots. The simple roots are a set of six complete, linearly independent root vectors from which the entire algebra can be constructed. Given two simple roots $v_{\alpha}, v_{\beta}$; the angle between them is calculated using the inner product,

$$
\cos \theta_{\alpha \beta}=\frac{\left\langle v_{\alpha}, v_{\beta}\right\rangle}{\left\|v _ { \alpha } \left|\left\|\mid v_{\beta}\right\|\right.\right.} .
$$

The angles between the simple roots take values of $\frac{\pi}{2}$ and $\frac{2 \pi}{3}$. Opening up the conversation to positive roots allows for angles of $\frac{\pi}{3}$, and the negative roots are obviously located at angles of $\pi$ from the corresponding positive root. A graphical representation of the root 
system can be found in Figure 1. Given two simple roots, $v_{\alpha}$ and $v_{\beta}$, the information about angles between them is encoded in the Cartan matrix of $\mathrm{SU}(7)$ with components defined by,

$$
A_{\alpha \beta}=2 \frac{\left\langle v_{\alpha}, v_{\beta}\right\rangle}{\left\langle v_{\alpha}, v_{\alpha}\right\rangle} .
$$

The Cartan matrix was calculated explicitly to be,

$$
A_{\alpha \beta}=\left(\begin{array}{cccccc}
2 & -1 & 0 & 0 & 0 & 0 \\
-1 & 2 & -1 & 0 & 0 & 0 \\
0 & -1 & 2 & -1 & 0 & 0 \\
0 & 0 & -1 & 2 & -1 & 0 \\
0 & 0 & 0 & -1 & 2 & -1 \\
0 & 0 & 0 & 0 & -1 & 2
\end{array}\right) .
$$

A zero entry in the $m, n$ position denotes an angle of $\frac{\pi}{2}$ between the $m$ and $n$th simple roots, a -1 denotes an angle of $\frac{2 \pi}{3}$, and a 2 on the diagonal is an angle of 0 between a simple root and itself. Each simple root is orthogonal to but one other root. This can be seen from the Dynkin diagram below in Figure 2, where each circle corresponds to a simple root. A line connecting two circles indicates that there is an angle of $\frac{2 \pi}{3}$ between the corresponding roots, and circles with no connecting lines are orthogonal roots.

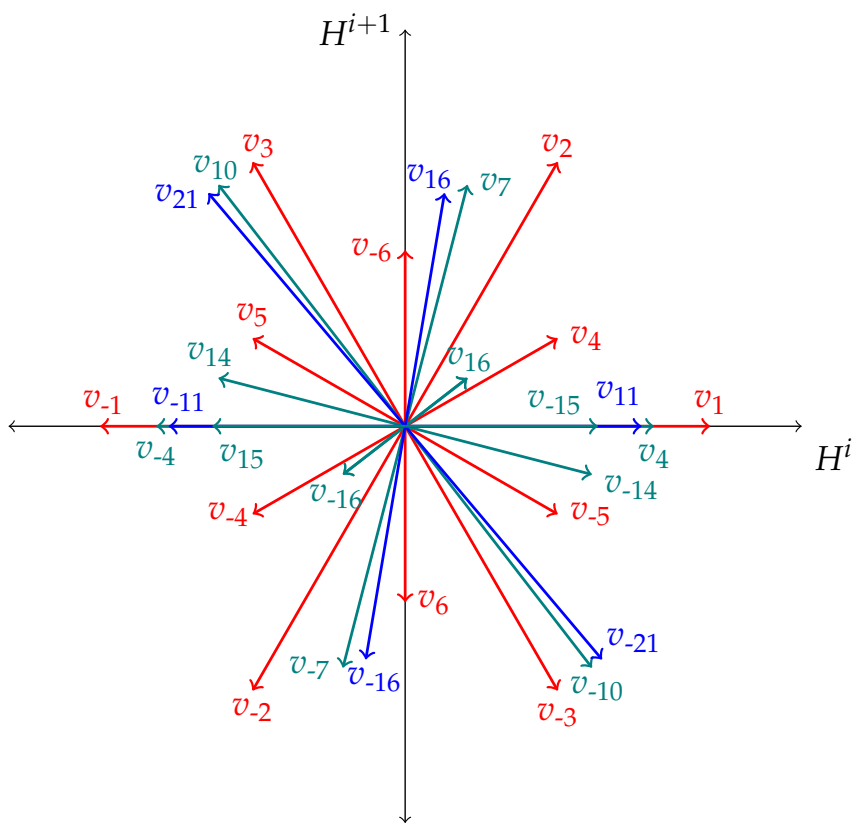

Figure 1. The root diagram for SU(7) plotted in the $H^{i}-H^{i+1}$ planes. Red, teal, and blue correspond to the planes in which $i=1,3,5$ respectively. Short roots correspond to roots that have components out of the corresponding plane. The short roots contain between 2 and 5 individual roots that differ only in extra-planar components. They are labeled with the first root characterized by these planar components.

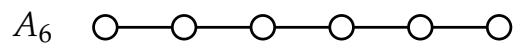

Figure 2. The Dynkin Diagram for su(7), a simple Lie algebra of rank 6.

Figure 1 shows the projection of the root system onto 2 dimensional planes. From this, some of the geometric properties discussed in the section are made visually apparent. The preservation of the $\mathrm{SU}(3)$ roots $\mathrm{I}_{ \pm}, \mathrm{V}_{ \pm}$, and $\mathrm{U}_{ \pm}$is seen in the red $H^{1}-H^{2}$ plane as $v_{ \pm 1}$, $v_{ \pm 2}$, and $v_{ \pm 3}$ respectively. The positive and simple roots have been explicitly calculated and can be found in Table A4 in Appendix A. 
The root vectors describe all possible movement between states in any given representation, and so describe interactions between particles. This becomes interesting as different particle compositions are explored, in which predictions such as proton decay arise. The gauge bosons mediating these interactions are housed in the adjoint representation, for which the roots serve as weights.

\section{Symmetry Breaking Patterns}

The issue at hand is that at high energy the $\mathrm{SU}(7)$ symmetry is a good symmetry but at lower energies there must be some symmetry breakdown. In a similar fashion as to the Standard Model where the Higgs mechanism is invoked in order to trigger (induce) the break the the electroweak $S U(2)_{L} \times U(1)_{Y}$ to $U(1)_{E M}$. This endows the $S U(2)$ weak bosons to acquire mass. In grand unified theories at least one additional symmetry breaking is required. In fact the $S U(7)$ model must break symmetry at several times in order to realize the observed Standard Model symmetries. There are numerous symmetry breaking chains in which this can be accomplished.

As discussed in Section 1, the symmetry breaking chain may be employed in order to play a role in cosmological applications that results in inflation, baryogenesis, the creation of heavy neutrinos, and other such phenomena. Various mass scales relate to phase transitions below the Planck scale, $M_{p l} \sim 10^{18} \mathrm{GeV}$, associated with the subsequent symmetry breaking chain from the GUT scale, $M_{G U T} \sim 10^{16} \mathrm{GeV}$ down to the electroweak scale, $M_{E M} \sim 10^{10^{2}} \mathrm{GeV}$.

The symmetry breaking chain may also be invoked on aesthetic grounds due to the extended high energy desert between the the electroweak scale and the GUT scale. Intermediate symmetry breaking, possibly associated with extended symmetries of unknown interactions, would populate this desert with various phenomena.

The chosen candidate gauge group for a GUT must be at least a rank 4 simple Lie group in order to have the Standard Model as a subgroup. The SU(7) gauge group of rank 6 offers model builders a great deal of freedom in choosing a number of different avenues to pursue symmetry breaking down $[43,44]$ to the non-simple gauge group of the Standard Model, $\mathrm{SU}(3)_{C} \times \mathrm{SU}(2)_{L} \times \mathrm{U}(1)_{Y} \rightarrow \mathrm{SU}(3)_{C} \times \mathrm{U}(1)_{\mathrm{em}}$. It should be noted that physical constraints should be imposed on the symmetry breaking chains such that the representations should be renormalizable and thus anomaly free along with respecting the the low energy effective theory representation of particles. A non-exhaustive list of some of theses symmetry group breaking patterns are,

$$
\begin{aligned}
& \mathrm{SU}(7) \rightarrow \mathrm{SO}(7), \\
& \mathrm{SU}(7) \rightarrow \mathrm{SU}(6) \times \mathrm{U}(1), \\
& \mathrm{SU}(7) \rightarrow \mathrm{SU}(5) \times \mathrm{SU}(2) \times \mathrm{U}(1), \\
& \mathrm{SU}(7) \rightarrow \mathrm{SU}(5) \times \mathrm{U}(1), \\
& \mathrm{SU}(7) \rightarrow \mathrm{SU}(4) \times \mathrm{SU}(3) \times \mathrm{U}(1),
\end{aligned}
$$

and

$$
\mathrm{SU}(7) \rightarrow \mathrm{SU}(2) \times \mathrm{SU}(2) \times \mathrm{SU}(3) \times \mathrm{U}(1) .
$$

A number of intermediate steps may be present in many of the symmetry breaking patterns described above.

This symmetry breaking is typically invoked by the Higgs mechanism [45-49]. The Lagrangian density associated with this scalar field, $\phi$, can be written as

$$
\mathcal{L}=-|\mathcal{D} \phi|^{2}+h \bar{F} \phi F-V(\phi)
$$


where the first term denotes the kinetic energy of the scalar field that includes the covariant derivative which describes the dynamics of the interaction, the second term are the fermionHiggs couplings and the third term is the Higgs potential.

The Higgs may transform under any number of representations. A few simple low dimensional potentials are given below. The scalar Higgs $\phi$ transforms according to the adjoint representation as

$$
V(\phi)=-\frac{1}{2} \mu^{2} \operatorname{Tr} \phi^{2}+\frac{a}{4}\left(\operatorname{Tr} \phi^{2}\right)^{2}+\frac{b}{2} \operatorname{Tr} \Phi^{4}+\frac{d}{3} \operatorname{Tr} \phi^{3}
$$

where $\mu^{2}, a, b$, and $c$ are constant parameters. The cubic $\phi^{3}$ coupling can be removed by imposing a $Z_{2}$ symmetry where $\phi \rightarrow-\phi$. The scalar Higgs $H$ transforms according to the fundamental representation as

$$
V(H)=-\frac{\mu_{f}^{2}}{2} H^{\dagger} H+\frac{a_{f}}{4}\left(H^{\dagger} H\right)^{2}
$$

where $\mu_{f}^{2}$ and $a_{f}$ are constant parameters. The potential associated with both representations contains the potentials along with a cross-term,

$$
V(\phi, H)=V(\phi)+V(H)+V(\phi, H)
$$

where the cross-term is given as

$$
V(\phi, H)=e H^{\dagger} H \operatorname{Tr} \phi^{2}+f H^{\dagger} \phi^{2} H+g H^{\dagger} \phi H
$$

where $e, f$, and $g$ are constant parameters.

It should be noted that the choice of representation of the Higgs field is somewhat arbitrary along with the fermion-Higgs coupling parameters. This leads to the particle masses and the spectrum not completely predicted by the model though relationships between the masses can sometimes be determined.

\section{A Review of Several Example SU(7) Models}

\subsection{Fractionally Charged Particles and SU(7) GUT Model}

Li and Wilczek [50] invoked an SU(7) symmetry in order to include fractionally charged leptonic or hadronic color singlets. In this model, they extended the symmetry group $\mathrm{SU}(5)$ to $\mathrm{SU}(7)$ by adding two extra states to the fundamental representation with opposite charges. The matter particles are assigned to the $7, \mathbf{3 5}, \overline{\mathbf{2 1}}$, and $\mathbf{1}$ representations. The charge assignment, $Q$, of the fundamental representation takes the form of,

$$
Q(7)=\operatorname{diag}\left[\begin{array}{lllllll}
\frac{1}{3} & \frac{1}{3} & \frac{1}{3} & -1 & 0 & q & -q
\end{array}\right]
$$

where $q$ is the electric charge of the postulated fractional charged color singlet particle. It should be noted that the hypercharge can be determined from the Gell-Mann-Nishijima formula [51-53]. This model allows for two neutral Majorana leptonic states which can have their masses generated via a see-saw mechanism.

The model builders consider a number of avenues for the symmetry breaking chain but with the

$$
\mathrm{SU}(7) \rightarrow \mathrm{SU}(4) \times \mathrm{SU}(3) \times \mathrm{U}(1) \rightarrow \mathrm{SU}(3) \times \mathrm{SU}(2) \times \mathrm{U}(1) \rightarrow \mathrm{SU}(3) \times \mathrm{U}(1)
$$

the prediction of the Weinberg angle with $\alpha=1 / 128$ and $\alpha_{S}=0.11$ is in agreement with the Georgi-Glashow prediction. In this model, if the grand unification scale is taken to be $10^{15} \mathrm{GeV}$ then the intermediate energy scale is of order $10^{9} \mathrm{GeV}$. The first stage of symmetry breaking is triggered by the Higgs in the $\mathbf{4 8}$ adjoint representation. The intermediate 
symmetry breaking stages can occur via several choices of the Higgs in the 21 and 756 representations, the $\mathbf{8 4 0}$ representation or the $\mathbf{8 4 0}$ and $\mathbf{7 5 6}$ representations with the final stage of symmetry breaking being performed with a Higgs in the fundamental representation.

\subsection{Dark Matter and SU(7) GUT Model}

Several model builders [54-56] invoked an SU(7) symmetry in order to explore asymmetric dark matter via the possibility of expanding the Standard Model to include a dark sector for dark matter particles and their associated dark gauge interactions. Focusing on the last two models, the GUT SU(7) symmetry is broken

$$
\mathrm{SU}(7) \rightarrow \mathrm{SU}(5)_{V} \times \mathrm{SU}(2)_{D} \times \mathrm{U}(1)
$$

into the visible $\mathrm{SU}(5)_{V}$ and the dark $\mathrm{SU}(2)_{D}$. The matter particles are assigned to the left-chiral representations: $\overline{7}, \mathbf{2 1}$, and $\overline{\mathbf{3 5}}$. In this model all the matter particles gain mass via the electroweak symmetry breaking. The model builders embed the electroweak Higgs doublet into the $\overline{7}$ representation in a similar fashion to the typical Georgi-Glashow SU(5) unification model. The issue with this straightforward application is that the left-handed neutrino-like particle will acquire a mass at the $\mathrm{MeV}$ scale which is in opposition to known experimental results. The $\mathbf{3 5}$ and tensor product of $\overline{\mathbf{3 5}} \times \overline{\mathbf{3 5}}$ are also considered. The final stage of symmetry breaking occurs via the 21 representation to break the $U(1)$ symmetry and acquire a vacuum expectation value. The limitations of this model do not end with maneuvering around the heavy left-handed like neutrino but continue to exists since the LHC has not observed such exotic particles at the TeV scale. Another drawback of this model is that the matter content of $\overline{\mathbf{7}} \oplus \mathbf{2 1} \oplus \overline{\mathbf{3 5}}$ produces two visible generations of the Standard Model. In order to include the whole Standard Model, this implies that there must be four generations of particles based upon the group theory but which the fourth generation of particles have not been observed.

\subsection{Supersymmetry and SU(7) GUT Model}

Numerous supersymmetric grand theories have been motivated to use the SU(7) symmetry group. Model builders Frampton and Kephart [57] and Kubo and Sakakibara [58] invoked this group in their own supersymmetric GUT studies of fractional charged particles based upon the Li and Wilczek non-susy GUT Model [50]. Besides expanding the Standard Model to include supersymmetry and fractionally charged particles, the model builders are also driven by the value of the Weinberg angle to agree with experiment and to reduce the ad hoc way in which many times light Higgs doublets must be added into the model. The model builders generalize the non-supersymmetric model with charge operator described above (see Equation (42)). The symmetry breaking chain is taken for simplicity to be

$$
\mathrm{SU}(7) \rightarrow \mathrm{SU}(3) \times \mathrm{SU}(2) \times \mathrm{U}(1) .
$$

It is noted that there is a possibility for extra $U(1)$ may be necessary. The values of the fractional charge given in Equation (42) are set to 1/3 along with requiring the number of light Higgs to be 2 in order to fix the one-loop renormalization group equation for $\sin ^{2} \theta_{W} \simeq 0.204$ at the weak scale. The issue with this model is if all the particle families are incorporated into this model then the gauge coupling of the strong interaction is greater than unity before unification and this leads to a non-perturbative QCD regime and where asymptotic freedom is not obeyed.

\section{Conclusions}

The raising and lowering operators for $\mathrm{SU}(7)$ have been constructed and the Cartan algebra has been identified. These form the Cartan-Weyl basis. The commutator relationship for the raising and lowering operators have been calculated. The roots have been tabulated along with the weights. The direct products of the fundamental and conjugate representations of $\mathrm{SU}(7)$ have been investigated by both tensorial and Young tableau 
methods. Dynkin diagrams and the Cartan matrix have been discussed. These results have been presented for $\mathrm{SU}(7)$ model builders to determine symmetry breaking chains along with their associated subgroups, branching rules to determine representation construction and decomposition, and other applications of these results. It should also be noted that many of these results can be utilized when examining the corresponding symmetry groups associated with the symmetry breaking of higher dimensional symmetry groups used in grand unification and string theory. The $\mathrm{SU}(7)$ group is highly utilized in string theory due to the fact that $\mathrm{SU}(7)$ is a subgroup of the E7 group.

It should be noted that besides the application of the $\mathrm{SU}(7)$ group to programs of grand unification, this group has also found a myriad of other applications in physics and mathematics where the above tabulations may be useful. The SU(7) group has been used widely in large- $N$ lattice QCD studies of glueball correlation functions [59], of the spectra and decay constants of mesons [60,61], and of critical deconfinement temperatures [62]. In quantum-chemistry and atomic physics, the issue at hand is inherently a many-body problem and by restricting the degrees of freedom for the Hamiltonian by using the $\mathrm{SU}(7)$ group allows for the calculation of selection rules [63,64]. This method has also been been applied to the quarks in nuclear physics [65] in an analogous manner.

Author Contributions: Conceptualization, J.A.S.; formal analysis, D.J.; investigation, D.J. and J.A.S.; writing, D.J. and J.A.S.; supervision, J.A.S. All authors have read and agreed to the published version of the manuscript.

Funding: This research received no external funding.

Institutional Review Board Statement: Not applicable.

Informed Consent Statement: Not applicable.

Data Availability Statement: Not applicable.

Acknowledgments: The authors would like to thank A. Goetz for many fruitful conversations and useful comments.

Conflicts of Interest: The authors declare no conflict of interest.

\section{Appendix A}

Appendix A.1. Generators

Table A1. The tabulated generators of SU(7).

\begin{tabular}{lll}
\hline & \multicolumn{1}{c}{ SU(7) Generators } & \\
\hline$\lambda_{1}=E_{12}+E_{21}$ & $\lambda_{2}=-i E_{12}+i E_{21}$ & $\lambda_{3}=E_{11}-E_{22}$ \\
$\lambda_{4}=E_{13}+E_{31}$ & $\lambda_{5}=-i E_{13}+i E_{31}$ & $\lambda_{6}=E_{23}+E_{32}$ \\
$\lambda_{7}=-i E_{23}+i E_{32}$ & $\lambda_{8}=\frac{1}{\sqrt{3}}\left(\sum_{k=1}^{2} E_{k k}-2 E_{33}\right)$ & $\lambda_{9}=E_{14}+E_{41}$ \\
$\lambda_{10}=-i E_{14}+i E_{41}$ & $\lambda_{11}=E_{24}+E_{42}$ & $\lambda_{12}=-i E_{24}+i E_{42}$ \\
$\lambda_{13}=E_{34}+E_{43}$ & $\lambda_{14}=-i E_{34}+i E_{43}$ & $\lambda_{15}=\frac{1}{\sqrt{6}}\left(\sum_{k=1}^{3} E_{k k}-3 E_{44}\right)$ \\
$\lambda_{16}=E_{15}+E_{51}$ & $\lambda_{17}=-i E_{15}+i E_{51}$ & $\lambda_{18}=E_{25}+E_{52}$ \\
$\lambda_{19}=-i E_{25}+i E_{52}$ & $\lambda_{20}=E_{35}+E_{53}$ & $\lambda_{21}=-i E_{35}+i E_{53}$ \\
$\lambda_{22}=E_{45}+E_{54}$ & $\lambda_{23}=-i E_{45}+i E_{54}$ & $\lambda_{24}=\frac{1}{\sqrt{10}}\left(\sum_{k=1}^{4} E_{k k}-4 E_{55}\right)$ \\
$\lambda_{25}=E_{16}+E_{61}$ & $\lambda_{26}=-i E_{16}+i E_{61}$ & $\lambda_{27}=E_{26}+E_{62}$ \\
$\lambda_{28}=-i E_{26}+i E_{62}$ & $\lambda_{29}=E_{36}+E_{63}$ & $\lambda_{30}=-i E_{36}+i E_{63}$ \\
$\lambda_{31}=E_{46}+E_{64}$ & $\lambda_{32}=-i E_{46}+i E_{64}$ & $\lambda_{33}=E_{56}+E_{65}$ \\
$\lambda_{34}=-i E_{56}+i E_{65}$ & $\lambda_{35}=\frac{1}{\sqrt{15}}\left(\sum_{k=1}^{5} E_{k k}-5 E_{66}\right)$ & $\lambda_{36}=E_{17}+E_{71}$ \\
$\lambda_{37}=-i E_{17}+i E_{71}$ & $\lambda_{38}=E_{27}+E_{72}$ & $\lambda_{39}=-i E_{27}+i E_{72}$ \\
$\lambda_{40}=E_{37}+E_{73}$ & $\lambda_{41}=-i E_{37}+i E_{73}$ & $\lambda_{42}=E_{47}+E_{74}$ \\
$\lambda_{43}=-i E_{47}+i E_{74}$ & $\lambda_{44}=E_{57}+E_{75}$ & $\lambda_{45}=-i E_{57}+i E_{75}$ \\
$\lambda_{46}=E_{67}+E_{76}$ & $\lambda_{47}=-i E_{67}+i E_{76}$ & $\lambda_{48}=\frac{1}{\sqrt{21}}\left(\sum_{k=1}^{6} E_{k k}-6 E_{77}\right)$ \\
\hline
\end{tabular}




\section{Appendix A.2. Symmetric Structure Functions}

Table A2. The anti-symmetric structure functions.

\begin{tabular}{|c|c|c|c|c|c|c|c|c|c|c|c|}
\hline \multicolumn{12}{|c|}{ The Anti-Symmetric Structure Functions of SU(7) } \\
\hline $\mathbf{i} \mathbf{j} \mathbf{k}$ & $f_{i j k}$ & $\mathbf{i} \mathbf{j} \mathbf{k}$ & $f_{i j k}$ & $\mathbf{i} \mathbf{j} \mathbf{k}$ & $f_{i j k}$ & $\mathbf{i} \mathbf{j} \mathbf{~ k}$ & $f_{i j k}$ & $\mathbf{i} \mathbf{j} \mathbf{k}$ & $f_{i j k}$ & $\mathbf{i} \mathbf{j} \mathbf{k}$ & $f_{i j k}$ \\
\hline 123 & 1 & 81112 & $\frac{\sqrt{3}}{6}$ & 101723 & $\frac{1}{2}$ & 52529 & $\frac{1}{2}$ & 162633 & $-\frac{1}{2}$ & 13639 & $\frac{1}{2}$ \\
\hline & & 81314 & $-\frac{\sqrt{3}}{3}$ & 111823 & $\frac{1}{2}$ & 52630 & $\frac{1}{2}$ & 172533 & $\frac{1}{2}$ & 13738 & $-\frac{1}{2}$ \\
\hline 147 & $\frac{1}{2}$ & 91015 & $\frac{\sqrt{6}^{0}}{3}$ & 111922 & $-\frac{1}{2}$ & 62730 & $\frac{1}{2}$ & 172634 & $\frac{1}{2}$ & 23638 & $\frac{1}{2}$ \\
\hline 156 & $-\frac{1}{2}$ & 111215 & $\frac{\sqrt[3]{6}}{3}$ & 121822 & $\frac{1}{2}$ & 62829 & $-\frac{1}{2}$ & 182734 & $\frac{1}{2}$ & 23739 & $\frac{1}{2}$ \\
\hline 246 & $\frac{1}{2}$ & 131415 & $\frac{\sqrt[3]{6}}{3}$ & 121923 & $\frac{1}{2}$ & 72729 & $\frac{1}{2}$ & 182833 & $-\frac{1}{2}$ & 33637 & $\frac{1}{2}$ \\
\hline 257 & $\frac{1}{2}$ & \multicolumn{2}{|c|}{$-4-^{3}$} & 132023 & $\frac{1}{2}$ & 72830 & $\frac{1}{2}$ & 192733 & $\frac{1}{2}$ & 33839 & $-\frac{1}{2}$ \\
\hline 345 & $\frac{1}{2}$ & 11619 & $\frac{1}{2}$ & 132122 & $-\frac{1}{2}$ & 82526 & $\frac{\sqrt{3}}{6}$ & 192834 & $\frac{1}{2}$ & 43641 & $\frac{1}{2}^{2}$ \\
\hline 367 & $-\frac{1}{2}$ & 11718 & $-\frac{1}{2}$ & 142022 & $\frac{1}{2}$ & 82728 & $\frac{\sqrt[6]{3}}{6}$ & 202934 & $\frac{1}{2}$ & 43740 & $-\frac{1}{2}$ \\
\hline 458 & $\frac{\sqrt{3}}{2}$ & 21618 & $\frac{1}{2}^{2}$ & 142123 & $\frac{1}{2}$ & 82930 & $-\frac{\sqrt{3}}{3}$ & 203033 & $-\frac{1}{2}$ & 53640 & $\frac{1}{2}$ \\
\hline 678 & $\frac{\sqrt{3}}{2}$ & 21719 & $\frac{1}{2}$ & 151617 & $\frac{\sqrt{6}}{12}$ & 92532 & $\frac{1}{2}^{3}$ & 212933 & $\frac{1}{2}$ & 53741 & $\frac{1}{2}$ \\
\hline & & 31617 & $\frac{1}{2}$ & 151819 & $\frac{12}{12}$ & 92631 & $-\frac{1}{2}$ & 213034 & $\frac{1}{2}$ & 63841 & $\frac{1}{2}$ \\
\hline 1912 & $\frac{1}{2}$ & 31819 & $-\frac{1}{2}$ & 152021 & $\frac{12}{\sqrt{6}}$ & 102531 & $\frac{1}{2}$ & 223134 & $\frac{1}{2}$ & 63940 & $-\frac{1}{2}$ \\
\hline 11011 & $-\frac{1}{2}$ & 41621 & $\frac{1}{2}^{2}$ & 152223 & $-\frac{\sqrt{6}}{4}$ & 102632 & $\frac{1}{2}$ & 223233 & $-\frac{1}{2}$ & 73840 & $\frac{1}{2}$ \\
\hline 2911 & $\frac{1}{2}$ & 41720 & $-\frac{1}{2}$ & 161724 & $\frac{\sqrt{10}}{4}$ & 112732 & $\frac{1}{2}$ & 233133 & $\frac{1}{2}$ & 73941 & $\frac{1}{2}$ \\
\hline 21012 & $\frac{1}{2}$ & 51620 & $\frac{1}{2}$ & 181924 & $\frac{\sqrt[4]{10}}{4}$ & 112831 & $-\frac{1}{2}$ & 233234 & $\frac{1}{2}$ & 83637 & $\frac{\sqrt{3}}{6}$ \\
\hline 3910 & $\frac{1}{2}$ & 51721 & $\frac{1}{2}$ & 202124 & $\frac{\sqrt[4]{10}}{4}$ & 122731 & $\frac{1}{2}$ & 242526 & $\frac{\sqrt{10}}{20}$ & 83839 & $\frac{\sqrt[6]{3}}{6}$ \\
\hline 31112 & $-\frac{1}{2}$ & 61821 & $\frac{1}{2}$ & 222324 & $\frac{\frac{4}{10}}{4}$ & 122832 & $\frac{1}{2}$ & 242728 & $\frac{\sqrt{10}}{20}$ & 84041 & $\begin{array}{l}6 \\
-\frac{\sqrt{3}}{3}\end{array}$ \\
\hline 4914 & $\frac{1}{2}$ & 61920 & $-\frac{1}{2}$ & \multicolumn{2}{|c|}{$-5-\overline{4}$} & 132932 & $\frac{1}{2}$ & 242930 & $\frac{\sqrt{10}}{20}$ & 93643 & $\frac{1}{2}$ \\
\hline 41013 & $-\frac{1}{2}$ & 71820 & $\frac{1}{2}$ & 12528 & $\frac{1}{2}$ & 133031 & $-\frac{1}{2}$ & 243132 & $\frac{\sqrt{10}}{20}$ & 93742 & $-\frac{1}{2}$ \\
\hline 5913 & $\frac{1}{2}$ & 71921 & $\frac{1}{2}$ & 12627 & $-\frac{1}{2}$ & 142931 & $\frac{1}{2}$ & 243334 & $-\frac{\sqrt{10}}{5}$ & 103642 & $\frac{1}{2}$ \\
\hline 51014 & $\frac{1}{2}$ & 81617 & $\frac{\sqrt{3}}{6}$ & 22527 & $\frac{1}{2}$ & 143032 & $\frac{1}{2}$ & 252635 & $\frac{\sqrt{15}}{5}$ & 103743 & $\frac{1}{2}$ \\
\hline 61114 & $\begin{array}{l}\frac{2}{2} \\
\frac{1}{2}\end{array}$ & 81819 & $\frac{6}{6}$ & 22628 & $\frac{2}{2}$ & 152526 & $\frac{\sqrt{6}}{12}$ & 272835 & $\frac{5}{\sqrt{15}}$ & 113843 & $\begin{array}{l}2 \\
\frac{1}{2}\end{array}$ \\
\hline 61213 & $-\frac{1}{2}$ & 82021 & $\begin{array}{l}6 \\
-\frac{\sqrt{3}}{3}\end{array}$ & 32526 & $\frac{1}{2}$ & 152728 & $\frac{12}{12}$ & 293035 & $\frac{\sqrt[5]{15}}{5}$ & 113942 & $-\frac{1}{2}$ \\
\hline 71113 & $\frac{1}{2}$ & 91623 & $\frac{1}{2}^{3}$ & 32728 & $-\frac{1}{2}$ & 152930 & $\frac{\sqrt{6}}{12}$ & 313235 & $\frac{\sqrt{15}}{5}$ & 123842 & $\frac{1}{2}$ \\
\hline 71214 & $\frac{1}{2}$ & 91722 & $-\frac{1}{2}$ & 42530 & $\frac{1}{2}$ & 153132 & $-\frac{\sqrt{6}}{4}$ & 333435 & $\frac{\sqrt{15}}{5}$ & 123943 & $\frac{1}{2}$ \\
\hline 8910 & $\frac{\sqrt{3}}{6}$ & 101622 & $\frac{1}{2}$ & 42629 & $-\frac{1}{2}$ & 162534 & $\frac{1}{2}^{4}$ & \multicolumn{2}{|c|}{$-6-^{5}$} & 134043 & $\frac{1}{2}$ \\
\hline 134142 & $-\frac{1}{2}$ & 183845 & $\frac{1}{2}$ & 234345 & $\frac{1}{2}$ & 273946 & $-\frac{1}{2}$ & 334447 & $\frac{1}{2}$ & 383948 & $\frac{\sqrt{21}}{6}$ \\
\hline 144042 & $\frac{1}{2}$ & 183944 & $-\frac{1}{2}$ & 243637 & $\frac{\sqrt{10}}{20}$ & 283846 & $\frac{1}{2}$ & 334546 & $-\frac{1}{2}$ & 404148 & $\frac{\sqrt{21}}{6}$ \\
\hline 144143 & $\frac{1}{2}$ & 193844 & $\frac{1}{2}$ & 243839 & $\frac{\sqrt{10}}{20}$ & 283947 & $\frac{1}{2}$ & 344446 & $\frac{1}{2}$ & 424348 & $\frac{\sqrt{21}}{6}$ \\
\hline 153637 & $\frac{\sqrt{6}}{12}$ & 193945 & $\frac{1}{2}$ & 244041 & $\frac{20}{20}$ & 294047 & $\begin{array}{l}2 \\
\frac{1}{2}\end{array}$ & 344547 & $\begin{array}{l}2 \\
\frac{1}{2}\end{array}$ & 444548 & $\frac{6}{\sqrt{21}}$ \\
\hline 153839 & $\frac{\sqrt{6}}{12}$ & 204045 & $\frac{1}{2}$ & 244243 & $\frac{2 \sqrt{10}}{20}$ & 294146 & $-\frac{1}{2}$ & 353637 & $\frac{\sqrt{15}}{30}$ & 464748 & $\frac{\sqrt{21}}{6}$ \\
\hline 154041 & $\frac{12}{\sqrt{6}}$ & 204144 & $-\frac{1}{2}$ & 244445 & $\begin{array}{r}20 \\
-\frac{\sqrt{10}}{5}\end{array}$ & 304046 & $\frac{1}{2}$ & 353839 & $\frac{\sqrt[30]{15}}{30}$ & \multicolumn{2}{|c|}{$-7-{ }^{6}$} \\
\hline 154243 & $-\frac{\sqrt{6}}{4}$ & 214044 & $\frac{1}{2}$ & 253647 & $\frac{1}{2}$ & 304147 & $\frac{1}{2}$ & 354041 & $\frac{30}{30}$ & & \\
\hline 163645 & $\frac{1}{2}$ & 214145 & $\frac{1}{2}$ & 253746 & $-\frac{1}{2}$ & 314247 & $\frac{1}{2}$ & 354243 & $\begin{array}{l}30 \\
\frac{\sqrt{15}}{30}\end{array}$ & & \\
\hline 163744 & $-\frac{1}{2}$ & 224245 & $\frac{1}{2}$ & 263646 & $\frac{1}{2}$ & 314346 & $-\frac{1}{2}$ & 354445 & $\frac{30}{15}$ & & \\
\hline 173644 & $\frac{1}{2}$ & 224344 & $-\frac{1}{2}$ & 263747 & $\frac{1}{2}$ & 324246 & $\frac{1}{2}$ & 354647 & $\begin{array}{r}30 \\
-\frac{\sqrt{15}}{6}\end{array}$ & & \\
\hline 173745 & $\frac{1}{2}$ & 234244 & $\frac{1}{2}^{2}$ & 273847 & $\frac{1}{2}$ & 324347 & $\frac{1}{2}$ & 363748 & $\frac{\sqrt{21}}{6}$ & & \\
\hline
\end{tabular}




\section{Appendix A.3. Raising and Lowering Operators}

Table A3. The tabulated raising and lowering operators of SU(7).

\begin{tabular}{lll}
\hline & \multicolumn{1}{c}{ Raising Operators } \\
\hline$V_{1}=\frac{1}{\sqrt{2}} E_{12}$ & $V_{2}=\frac{1}{\sqrt{2}} E_{13}$ & $V_{3}=\frac{1}{\sqrt{2}} E_{23}$ \\
$V_{4}=\frac{1}{\sqrt{2}} E_{14}$ & $V_{5}=\frac{1}{\sqrt{2}} E_{24}$ & $V_{6}=\frac{1}{\sqrt{2}} E_{34}$ \\
$V_{7}=\frac{1}{\sqrt{2}} E_{15}$ & $V_{8}=\frac{1}{\sqrt{2}} E_{25}$ & $V_{9}=\frac{1}{\sqrt{2}} E_{35}$ \\
$V_{10}=\frac{1}{\sqrt{2}} E_{45}$ & $V_{11}=\frac{1}{\sqrt{2}} E_{16}$ & $V_{12}=\frac{1}{\sqrt{2}} E_{26}$ \\
$V_{13}=\frac{1}{\sqrt{2}} E_{36}$ & $V_{14}=\frac{1}{\sqrt{2}} E_{46}$ & $V_{15}=\frac{1}{\sqrt{2}} E_{56}$ \\
$V_{16}=\frac{1}{\sqrt{2}} E_{17}$ & $V_{17}=\frac{1}{\sqrt{2}} E_{27}$ & $V_{18}=\frac{1}{\sqrt{2}} E_{37}$ \\
$V_{19}=\frac{1}{\sqrt{2}} E_{47}$ & $V_{20}=\frac{1}{\sqrt{2}} E_{57}$ & $V_{21}=\frac{1}{\sqrt{2}} E_{67}$ \\
\hline & \multicolumn{1}{c}{ Lowering Operators } & \\
\hline$V_{-1}=\frac{1}{\sqrt{2}} E_{21}$ & $V_{-2}=\frac{1}{\sqrt{2}} E_{31}$ & $V_{-3}=\frac{1}{\sqrt{2}} E_{32}$ \\
$V_{-4}=\frac{1}{\sqrt{2}} E_{41}$ & $V_{-5}=\frac{1}{\sqrt{2}} E_{42}$ & $V_{-6}=\frac{1}{\sqrt{2}} E_{43}$ \\
$V_{-7}=\frac{1}{\sqrt{2}} E_{51}$ & $V_{-8}=\frac{1}{\sqrt{2}} E_{52}$ & $V_{-12}=\frac{1}{\sqrt{2}} E_{53}$ \\
$V_{-10}=\frac{1}{\sqrt{2}} E_{54}$ & $V_{-11}=\frac{1}{\sqrt{2}} E_{61}$ & $V_{-15}=\frac{1}{\sqrt{2}} E_{65}$ \\
$V_{-13}=\frac{1}{\sqrt{2}} E_{63}$ & $V_{-14}=\frac{1}{\sqrt{2}} E_{64}$ & $V_{-18}=\frac{1}{\sqrt{2}} E_{73}$ \\
$V_{-16}=\frac{1}{\sqrt{2}} E_{71}$ & $V_{-17}=\frac{1}{\sqrt{2}} E_{72}$ & $V_{-21}=\frac{1}{\sqrt{2}} E_{76}$ \\
$V_{-19}=\frac{1}{\sqrt{2}} E_{74}$ & $V_{-20}=\frac{1}{\sqrt{2}} E_{75}$ &
\end{tabular}

Appendix A.4. Positive and Simple Roots

Table A4. The calculated values of the positive and simple roots of SU(7).

\begin{tabular}{ll}
\hline \multicolumn{1}{c}{ Positive Roots } \\
\hline$v_{1}=(1,0,0,0,0,0)$ & $v_{2}=\left(\frac{1}{2}, \frac{\sqrt{3}}{2}, 0,0,0,0\right)$ \\
$v_{-3}=\left(\frac{1}{2},-\frac{\sqrt{3}}{2}, 0,0,0,0\right)$ & $v_{4}=\left(\frac{1}{2}, \frac{\sqrt{3}}{6}, \frac{\sqrt{6}}{3}, 0,0,0\right)$ \\
$v_{-5}=\left(\frac{1}{2},-\frac{\sqrt{3}}{6},-\frac{\sqrt{6}}{3}, 0,0,0\right)$ & $v_{-6}=\left(0, \frac{\sqrt{3}}{3},-\frac{\sqrt{6}}{3}, 0,0,0\right)$ \\
$v_{7}=\left(\frac{1}{2}, \frac{\sqrt{3}}{6}, \frac{\sqrt{6}}{12}, \frac{\sqrt{10}}{4}, 0,0\right)$ & $v_{-8}=\left(\frac{1}{2},-\frac{\sqrt{3}}{6},-\frac{\sqrt{6}}{12},-\frac{\sqrt{10}}{4}, 0,0\right)$ \\
$v_{-9}=\left(0, \frac{\sqrt{3}}{3},-\frac{\sqrt{6}}{12},-\frac{\sqrt{10}}{4}, 0,0\right)$ & $v_{-10}=\left(0,0, \frac{\sqrt{6}}{4},-\frac{\sqrt{10}}{4}, 0,0\right)$ \\
$v_{11}=\left(\frac{1}{2}, \frac{\sqrt{3}}{6}, \frac{\sqrt{6}}{12}, \frac{\sqrt{10}}{20}, \frac{\sqrt{15}}{5}, 0\right)$ & $v_{-12}=\left(\frac{1}{2},-\frac{\sqrt{3}}{6},-\frac{\sqrt{6}}{12},-\frac{\sqrt{10}}{20},-\frac{\sqrt{15}}{5}, 0\right)$ \\
$v_{-13}=\left(0, \frac{\sqrt{3}}{3},-\frac{\sqrt{6}}{12},-\frac{\sqrt{10}}{20},-\frac{\sqrt{15}}{5}, 0\right)$ & $v_{-14}=\left(0,0, \frac{\sqrt{6}}{4},-\frac{\sqrt{10}}{20},-\frac{\sqrt{15}}{5}, 0\right)$ \\
$v_{-15}=\left(0,0,0, \frac{\sqrt{10}}{5},-\frac{\sqrt{15}}{5}, 0\right)$ & $v_{16}=\left(\frac{1}{2}, \frac{\sqrt{3}}{6}, \frac{\sqrt{6}}{12}, \frac{\sqrt{10}}{20}, \frac{\sqrt{15}}{30}, \frac{\sqrt{21}}{6}\right)$ \\
$v_{-17}=\left(\frac{1}{2},-\frac{\sqrt{3}}{6},-\frac{\sqrt{6}}{12},-\frac{\sqrt{10}}{20},-\frac{\sqrt{15}}{30},-\frac{\sqrt{21}}{6}\right)$ & $v_{-18}=\left(0, \frac{\sqrt{3}}{3},-\frac{\sqrt{6}}{12},-\frac{\sqrt{10}}{20},-\frac{\sqrt{15}}{30},-\frac{\sqrt{21}}{6}\right)$ \\
$v_{-19}=\left(0,0, \frac{\sqrt{6}}{4},-\frac{\sqrt{10}}{20},-\frac{\sqrt{15}}{30},-\frac{\sqrt{21}}{6}\right)$ & $v_{-20}=\left(0,0,0, \frac{\sqrt{10}}{5},-\frac{\sqrt{15}}{30},-\frac{\sqrt{21}}{6}\right)$ \\
$v_{-21}=\left(0,0,0,0, \frac{\sqrt{15}}{6},-\frac{\sqrt{21}}{6}\right)$ & \\
\hline & Simple Roots \\
\hline$v_{-3}=\left(\frac{1}{2},-\frac{\sqrt{3}}{2}, 0,0,0,0\right)$ & $v_{-6}=\left(0, \frac{\sqrt{3}}{3},-\frac{\sqrt{6}}{3}, 0,0,0\right)$ \\
$v_{-10}=\left(0,0, \frac{\sqrt{6}}{4},-\frac{\sqrt{10}}{4}, 0,0\right)$ & $v_{-15}=\left(0,0,0, \frac{\sqrt{10}}{5},-\frac{\sqrt{15}}{5}, 0\right)$ \\
$v_{-21}=\left(0,0,0,0, \frac{\sqrt{15}}{6},-\frac{\sqrt{21}}{6}\right)$ & $v_{16}=\left(\frac{1}{2}, \frac{\sqrt{3}}{6}, \frac{\sqrt{6}}{12}, \frac{\sqrt{10}}{20}, \frac{\sqrt{15}}{30}, \frac{\sqrt{21}}{6}\right)$ \\
\hline
\end{tabular}




\section{Appendix A.5. Cartan Generators}

Table A5. The tabulated Cartan generators of SU(7).

\begin{tabular}{lc}
\hline & Cartan Generators \\
\hline$H^{1}=\frac{1}{2}\left[E_{11}-E_{22}\right]$ & $H^{2}=\frac{1}{2}\left[\sum_{i=1}^{2} E_{i i}-2 E_{33}\right]$ \\
$H^{3}=\frac{1}{2}\left[\sum_{i=1}^{3} E_{i i}-3 E_{44}\right]$ & $H^{4}=\frac{1}{2}\left[\sum_{i=1}^{4} E_{i i}-4 E_{55}\right]$ \\
$H^{5}=\frac{1}{2}\left[\sum_{i=1}^{5} E_{i i}-5 E_{66}\right]$ & $H^{6}=\frac{1}{2}\left[\sum_{i=1}^{6} E_{i i}-6 E_{77}\right]$ \\
\hline
\end{tabular}

\section{References}

1. Glashow, S.L. Partial-symmetries of weak interactions. Nucl. Phys. 1961, 22, 579-588. [CrossRef]

2. Gross, D.J.; Wilczek, F. Ultraviolet Behavior of Non-Abelian Gauge Theories. Phys. Rev. Lett. 1973, 30, 1343-1346. [CrossRef]

3. Salam, A.; Ward, J.C. Electromagnetic and weak interactions. Phys. Lett. 1964, 13, 168-171. [CrossRef]

4. Salam, A. Weak and Electromagnetic Interactions. Conf. Proc. C 1968, 680519, 367-377. [CrossRef]

5. Weinberg, S. A Model of Leptons. Phys. Rev. Lett. 1967, 19, 1264-1266. [CrossRef]

6. Weinberg, S. Non-Abelian Gauge Theories of the Strong Interactions. Phys. Rev. Lett. 1973, 31, 494-497. [CrossRef]

7. Yang, C.N.; Mills, R.L. Conservation of Isotopic Spin and Isotopic Gauge Invariance. Phys. Rev. 1954, 96, 191-195. [CrossRef]

8. Prescott, C.; Atwood, W.B.; Cottrell, R.L.A.; Destaebler, H.; Garwin, E.L.; Gonidec, A.; Miller, R.H.; Rochester, L.S.; Sato, T.; Sherden, D.J.; et al. Parity Nonconservation in Inelastic Electron Scattering. Phys. Lett. B 1978, 77, 347-352. [CrossRef]

9. Prescott, C.; Atwood, W.; Cottrell, R.; DeStaebler, H.; Garwin, E.L.; Gonidec, A.; Miller, R.; Rochester, L.; Sato, T.; Sherden, D.; et al. Further measurements of parity non-conservation in inelastic electron scattering. Phys. Lett. B 1979, 84, 524-528. [CrossRef]

10. Hasert, F.; Kabe, S.; Krenz, W.; Von Krogh, J.; Lanske, D.; Morfin, J.; Schultze, K.; Weerts, H.; Bertrand-Coremans, G.; Sacton, J.; et al. Observation of Neutrino Like Interactions without Muon or Electron in the Gargamelle Neutrino Experiment. Phys. Lett. B 1973, 46, 138-140. [CrossRef]

11. Hasert, F.J.; Faissner, H.; Krenz, W.; Von Krogh, J.; Lanske, D. Search for elastic muon neutrino electron scattering. Phys. Lett. B 1973, 46, 121-124. [CrossRef]

12. Aad, G.; Abajyan, T.; Abbott, B.; Abdallah, J.; Khalek, S.A.; Abdelalim, A.A.; Abdinov, O.; Aben, R.; Abi, B.; Abolins, M.; et al. Observation of a new particle in the search for the Standard Model Higgs boson with the ATLAS detector at the LHC. Phys. Lett. B 2012, 716, 1-29. [CrossRef]

13. Aad, G.; Abajyan, T.; Abbott, B.; Abdallah, J.; Abdel Khalek, S.; Abdinov, O.; Aben, R.; Abi, B.; Abolins, M.; AbouZeid, O.S.; et al. Measurements of Higgs boson production and couplings in diboson final states with the ATLAS detector at the LHC. Phys. Lett. B 2013, 726, 88-119. [CrossRef]

14. Georgi, H.; Glashow, S.L. Unity of All Elementary Particle Forces. Phys. Rev. Lett. 1974, 32, 438-441. [CrossRef]

15. Pati, J.C.; Salam, A. Lepton Number as the Fourth Color. Phys. Rev. D 1974, 10, 275-289; Erratum in Phys. Rev. D 1975, $11,703$. [CrossRef]

16. Langacker, P. Grand Unified Theories and Proton Decay. Phys. Rept. 1981, 72, 185. [CrossRef]

17. Slansky, R. Group Theory for Unified Model Building. Phys. Rept. 1981, 79, 1-128. [CrossRef]

18. Dimopoulos, S.; Raby, S.; Wilczek, F. Supersymmetry and the Scale of Unification. Phys. Rev. D 1981, 24, 1681-1683. [CrossRef]

19. Guth, A.H. Inflationary universe: A possible solution to the horizon and flatness problems. Phys. Rev. D 1981, 23, 347-356. [CrossRef]

20. Linde, A. A new inflationary universe scenario: A possible solution of the horizon, flatness, homogeneity, isotropy and primordial monopole problems. Phys. Lett. B 1982, 108, 389-393. [CrossRef]

21. Albrecht, A.; Steinhardt, P.J. Cosmology for Grand Unified Theories with Radiatively Induced Symmetry Breaking. Phys. Rev. Lett. 1982, 48, 1220-1223. [CrossRef]

22. Efstathiou, G.P.; Ellis, R.S.; Gunn, J.E.; York, D.; Turner, M.S. Large-scale structure from quantum fluctuations in the early universe. Philos. Trans. R. Soc. Lond. Ser. A Math. Phys. Eng. Sci. 1999, 357, 7-20. [CrossRef]

23. Dvali, G.; Shafi, Q.; Schaefer, R. Large Scale Structure and Supersymmetric Inflation without Fine Tuning. Phys. Rev. Lett. 1994, 73, 1886-1889. [CrossRef] [PubMed]

24. Salopek, D.S.; Bond, J.R.; Bardeen, J.M. Designing density fluctuation spectra in inflation. Phys. Rev. D 1989, 40, 1753-1788. [CrossRef] [PubMed]

25. Di Marco, A.; Pradisi, G.; Cabella, P. Inflationary scale, reheating scale, and pre-BBN cosmology with scalar fields. Phys. Rev. D 2018, 98, 123511. [CrossRef]

26. Kolb, E.W.; Linde, A.; Riotto, A. Grand-Unified-Theory Baryogenesis after Preheating. Phys. Rev. Lett. 1996, 77, 4290-4293. [CrossRef]

27. Kofman, L.; Linde, A.; Starobinsky, A.A. Reheating after Inflation. Phys. Rev. Lett. 1994, 73, 3195-3198. [CrossRef]

28. Felder, G.N.; Kofman, L.; Linde, A.D. Instant preheating. Phys. Rev. D 1999, 59, 123523. [CrossRef]

29. Rangarajan, R.; Nanopoulos, D.V. Inflationary baryogenesis. Phys. Rev. D 2001, 64, 063511. [CrossRef] 
30. Kim, J.E. Flavor unity in SU (7): Low-mass magnetic monopole, doubly charged lepton, and Q= 53,- 43 quarks. Phys. Rev. D 1981, 23, 2706. [CrossRef]

31. Farhi, E.; Susskind, L. A TECHNICOLORED G.U.T. Phys. Rev. D 1979, 20, 202.

32. Farhi, E.; Susskind, L. Technicolour. Phys. Rep. 1981, 74, 277-321. [CrossRef]

33. Kuang, Y.P.; Tye, S.H. Grand unification with a confining model of the weak interactions. Phys. Rev. D 1982, 26, 1718. [CrossRef]

34. Chávez, H.; Martins Simões, J.A. The muon g-2 in an SU(7) left right symmetric model with mirror fermions. Nucl. Phys. B 2007, 783, 76-89. [CrossRef]

35. Diaz, R.A.; Gallego, D.; Martinez, R. Renormalization Group and Grand Unification with 331 Models. Int. J. Mod. Phys. A 2007, 22, 1849-1874. [CrossRef]

36. Brümmer, F.; Fichet, S.; Hebecker, A.; Kraml, S. Phenomenology of supersymmetric Gauge-Higgs unification. J. High Energy Phys. 2009, 2009, 011. [CrossRef]

37. Barr, S.M. Doubly lopsided mass matrices from supersymmetric $S U(N)$ unification. Phys. Rev. D 2008, 78, 055008. [CrossRef]

38. Goetz, A.; Secrest, J.A. The algebraic structure of the SU(7) Lie group. J. Math. Phys. 2019, 60, 101703. [CrossRef]

39. Group, P.D. Review of Particle Physics. Prog. Theor. Exp. Phys. 2020, 2020, 083C01, Available online: https://academic.oup.com/ ptep/article-pdf/2020/8/083C01/34673722/ptaa104.pdf (accessed on 5 April 2021). [CrossRef]

40. Pauli, W., Jr. Zur Quantenmechanik des magnetischen Elektrons. (On the quantum mechanics of the magnetic electron). Z. Phys. 1927, 43, 601-623. (In German) [CrossRef]

41. Gell-Mann, M. Symmetries of Baryons and Mesons. Phys. Rev. 1962, 125, 1067-1084. [CrossRef]

42. Einstein, A. Die Grundlage der allgemeinen Relativitätstheorie. Ann. Phys. 1916, 354, 769-822. [CrossRef]

43. Umemura, I.; Yamamoto, K. A Symmetry Breaking Pattern in the SU(7) Grand Unified Model. Prog. Theor. Phys. 1981, 66, 1430-1447. [CrossRef]

44. Feger, R.; Kephart, T.W.; Saskowski, R.J. LieART 2.0-A Mathematica application for Lie Algebras and Representation Theory. Comput. Phys. Commun. 2020, 257, 107490. [CrossRef]

45. Higgs, P.W. Broken symmetries, massless particles and gauge fields. Phys. Lett. 1964, 12, 132-133. [CrossRef]

46. Higgs, P.W. Broken Symmetries and the Masses of Gauge Bosons. Phys. Rev. Lett. 1964, 13, 508-509. [CrossRef]

47. Higgs, P.W. Spontaneous Symmetry Breakdown without Massless Bosons. Phys. Rev. 1966, 145, 1156-1163. [CrossRef]

48. Englert, F.; Brout, R. Broken Symmetry and the Mass of Gauge Vector Mesons. Phys. Rev. Lett. 1964, 13, 321-323. [CrossRef]

49. Guralnik, G.S.; Hagen, C.R.; Kibble, T.W.B. Global Conservation Laws and Massless Particles. Phys. Rev. Lett. 1964, 13, 585-587. [CrossRef]

50. Li, L.F.; Wilczek, F. Price of fractionally charged particles in a unified model. Phys. Lett. B 1981, 107, 64-68. [CrossRef]

51. Nakano, T.; Nishijima, K. Charge Independence for V-particles. Prog. Theor. Phys. 1953, 10, 581-582. [CrossRef]

52. Nishijima, K. Charge Independence Theory of V Particles. Prog. Theor. Phys. 1955, 13, 285-304. [CrossRef]

53. Gell-Mann, M. The interpretation of the new particles as displaced charge multiplets. Nuovo Cim. 1956, 4, 848-866. [CrossRef]

54. Ma, E. Unified framework for matter, dark matter, and radiative neutrino mass. Phys. Rev. D 2013, 88, 117702. [CrossRef]

55. Callen, B.D.; Volkas, R.R. Clash-of-symmetries mechanism from intersecting domain-wall branes. Phys. Rev. D 2014, 89, 056004. [CrossRef]

56. Callen, B.D. Unified Dark Matter from a Simple Gauge Group on a Domain-Wall Brane. arXiv 2017, arXiv:1705.02086.

57. Frampton, P.H.; Kephart, T.W. Fractionally Charged Particles as Evidence for Supersymmetry. Phys. Rev. Lett. 1982, 49, 1310-1313. [CrossRef]

58. Kubo, J.; Sakakibara, S. Spontaneous gauge symmetry breaking in nonminimal supersymmetric grand unified theories. J. Phys. G Nucl. Phys. 1983, 9, 475-496. [CrossRef]

59. Amato, A.; Bali, G.; Lucini, B. Topology and glueballs in SU(7) Yang-Mills with open boundary conditions. arXiv 2015. arXiv:1512.00806.

60. DeGrand, T.; Liu, Y.; Neil, E.T.; Shamir, Y.; Svetitsky, B. Spectroscopy of SU(4) gauge theory with two flavors of sextet fermions. Phys. Rev. D 2015, 91, 114502. [CrossRef]

61. Bali, G.; Bursa, F.; Castagnini, L.; Collins, S.; Del Debbio, L.; Lucini, B.; Panero, M. Mesons in large-N QCD. J. High Energy Phys. 2013, 2013. [CrossRef]

62. Lucini, B.; Rago, A.; Rinaldi, E. SU(Nc) gauge theories at deconfinement. Phys. Lett. B 2012, 712, 279-283. 2012.04.070. [CrossRef]

63. Racah, G. Theory of Complex Spectra. IV. Phys. Rev. 1949, 76, 1352-1365. [CrossRef]

64. Judd, B. Lie groups for atomic shells. Phys. Rep. 1997, 285, 1-76. [CrossRef]

65. Judd, B.R.; Lister, G.M.S. Complementary groups in the quark model of the atom. J. Phys. A Math. Gen. 1992, 25, 2615-2630. [CrossRef] 